\title{
INVESTIGAÇÃO DOS EFEITOS DO ÁCIDO 2,4 DICLOROFENOXIACÉTICO SOBRE DIFERENTES POPULAÇÕES DE NEURÔNIOS MIOENTÉRICOS DO DUODENO DE RATOS
}

\author{
Nathália Alves Diamante ${ }^{1}$ \\ Aline Aparecida Ribeiro ${ }^{2}$ \\ Vanessa Graciele Tibúrcio ${ }^{3}$ \\ Amanda Flávia da Silva Rovida ${ }^{4}$ \\ Renata de Britto Mari ${ }^{5}$ \\ Sandra Regina Stabille ${ }^{6}$ \\ Ricardo de Melo Germano ${ }^{7 *}$
}

\begin{abstract}
DIAMANTE, N. A.; RIBEIRO, A. A.; TIBÚRCIO, V. G.; ROVIDA, A. F. S.; MARI, R. de B.; STABILLE, S. R.; GERMANO, R. M. Investigação dos efeitos do ácido 2,4 diclorofenoxiacético sobre diferentes populações de neurônios mioentéricos do duodeno de ratos. Arq. Ciênc. Vet. Zool. UNIPAR, Umuarama, v. 17, n. 2, p. 97-105, abr./jun. 2014.
\end{abstract}

\begin{abstract}
RESUMO: O herbicida mais usado, tanto em pequenas como em grandes propriedades, por isso mais amplamente estudado é o 2,4-D. Os estudos de toxicidade têm se concentrado sobre as alterações do sistema nervoso central, e por isto pouco se conhece sobre seus efeitos no sistema nervoso entérico. Com o objetivo de avaliar os efeitos do 2,4-D sobre os neurônios mioentéricos do duodeno de ratos foi fornecido durante 60 dias doses de 2,4-D na concentração de $5 \mathrm{mg} / \mathrm{kg}$ de peso de corpóreo para ratosWistarde dois grupos experimentais $(n=5)$. Os animais dos grupos controle permaneceram o mesmo período sem receber 2,4-D. Ao final do período experimental os animais foram mortos, os duodenos foram coletados e processados por meio das técnicas histoquímicas de NADH-diaforase e NADPH-diaforase. Os neurônios foram quantificados e os resultados foram analisados estatisticamente. A densidade dos neurônios NADHd diferiu estatisticamente $(\mathrm{P}<0,05)$ entre os grupos experimental e controle, sendo maior no grupo controle. Já os neurônios NADPHd foram encontrados em maior quantidade no grupo experimental. Estes resultados sugerem que o 2,4-D possui ação neurotóxica sobre os neurônios do plexo mioentérico, interferindo na densidade neuronal mioentérica, quando se compara diferentes populações destes neurônios.
\end{abstract}

PALAVRAS-CHAVE: Plexomioentérico. Plasticidade neuronal. Intestino delgado. Herbicidas.

\section{INVESTIGATION OF 2,4 DICHLOROPHENOXYACETIC ACID EFFECTS IN DIFFERENT POPULATION OF RAT DUODENUM MYENTERIC NEURONS}

\begin{abstract}
The 2,4-D herbicide is the most widely used, both in small and in large properties. Therefore, it is also the one that is most broadly studied. Toxicity studies have been focused on changes in the central nervous system, and for this reason, little is known about its effect in the enteric nervous system. With the objective of measuring the effects of 2,4-D on the myenteric neurons in the duodenum of rats, doses of 2,4-D were supplied for 60 days at a concentration of $5 \mathrm{mg} / \mathrm{kg}$ of body weight to Wistar rats divided into two different experimental groups $(n=5)$. The animals in the control groups remained without 2,4-D doses for the same period. At the end of the experimental period, the animals were euthanized and their duodenum were collected and processed through NADH-diaphorase and NADPH-diaphorase histochemical techniques. The neurons were quantified and the results were statistically analyzed. The density of NADHd neurons differed statistically $(\mathrm{P}<0,05)$ between the experimental and control groups, being higher in the control group. However, NADPHd neurons were found in a greater quantity in the experimental group. These results suggest that the 2,4-D has a neurotoxic action in the neurons from the myenteric plexus, interfering in the myenteric neuronal density, when different population of these neurons are compared. KEYWORDS: Myenteric plexus. Neuronal plasticity. Small intestine. Herbicide.

\section{INVESTIGACIÓN DE LOS EFECTOS DEL ÁCIDO 2,4 DICLOROFENOXIACÉTICO SOBRE DIFERENTES POBLACIONES DE NEURONAS MIOENTÉRICAS DEL DUODENO DE RATONES}

RESUMEN: El herbicida más utilizado, tanto en pequeñas como en grandes propiedades es el 2,4-D, por eso lo más estudiado. Los estudios de toxicidad se han centrado sobre las alteraciones del sistema nervioso central, y por lo tanto, poco se sabe sobre sus efectos en el sistema nervioso entérico. Con el objetivo de evaluar los efectos del 2,4-D sobre las neuronas mioentéricas del duodeno de ratas se ha administrado durante 60 días dosis de 2,4-D en la concentración de $5 \mathrm{mg} / \mathrm{kg}$ de peso corporal para ratones Wistarde, dos grupos experimentales $(n=5)$. Los animales de los grupos control permanecieron el

\footnotetext{
DOI: https://doi.org/10.25110/arqvet.v17i2.2014.4926

${ }^{1}$ Bióloga - Bolsita do PEBIC/Fundação Araucária; Discente do Mestrado em Biologia Comparada - UEM. nathaliadiamante@gmail.com

${ }^{2}$ Bióloga - Discente do Mestrado em Biologia Comparada - UEM. alineribeiropvai@gmail.com

${ }^{3}$ Bióloga - Discente do Mestrado em Biologia Comparada - UEM. vanessinha.g7@hotmail.com

${ }^{4}$ Bióloga - Discente do Mestrado em Biotecnologia Ambiental - UEM. amanda_rovida@hotmail.com

${ }^{5}$ Professora Doutora da Universidade Estadual Paulista - UNESP - Câmpus Experimental do Litoral. Paulistaremari@clp.unesp.br

${ }^{6}$ Professora Doutora convidada do Departamento de Ciências Morfológicas - UEM. profasandra.regina@gmail.com

${ }^{7}$ Professor Doutor do Curso de Ciências Biológicas, Farmácia e do Mestrado em Ciência Animal da Universidade Paranaense - UNIPAR - Avenida Humberto Bruning, 360 - Jardim Santos Dumont - Paranavaí - PR, 87706-140, germano@unipar.br - * Autor para correspondência.
} 
mismo período sin recibir el 2,4-D. Al final del periodo experimental los animales fueron sacrificados, los duodenos fueron recogidos y procesados a través de las técnicas de histoquímicas de NADH-diaforasa y NADPH-diaforasa. Las neuronas fueron cuantificadas y los resultados analizados estadísticamente. La densidad de las neuronas NADHd difirió estadísticamente $(\mathrm{P}<0,05)$ entre los grupos experimental y control, siendo mayor en el grupo control. Ya las neuronas NADPHd se encontraron en mayor cantidad en el grupo experimental. Estos resultados sugieren que el 2,4-D tiene una acción neurotóxica sobre las neuronas del plexo mioentérico, lo que interfiere en la densidad neuronal mioentérica al comparar diferentes poblaciones de estas neuronas.

PALABRAS CLAVE: Plexo mioentérico. Plasticidad neuronal. Intestino delgado. Herbicidas.

\section{Introdução}

Os agrotóxicos estão entre os principais fatores de riscos para a saúde dos trabalhadores e ao meio ambiente (BRASIL, 2006).Os herbicidas ácidos são uma importante classe de agrotóxicos dos quais se destaca o ácido 2,4 diclorofenoxiacético $(2,4-\mathrm{D})$ por sua grande utilização em todo mundo (RODRIGUES; SERRA, 1996).

De amplo espectro no controle de ervas perenes e de folhas largas, em culturas de milho, cana-de-açúcar e pastagens,o 2,4-D é um herbicida sistêmico que atua como hormônio regulador do crescimento das plantas (EPA, 2005). Macroscopicamente, caracteriza-se pela apresentação na forma de um pó branco de odor levemente fenólico (TSMAN, 1991).Recomenda-se cuidado na aplicação, evitando períodos de vento, que mesmo fracos podem espalhar vapores do 2,4-D por longas distâncias (ALMEIDA; RODRIGUES, 1988).

Com a utilização de aproximadamente nove mil toneladas por ano o 2,4-D é o segundo herbicida mais utilizado no Brasil (WAITEet al., 2002; BERNARD et al., 2005),sendo encontrado em mais de 600 produtos (EPA, 2005), no entanto, no Brasil apenas 17 estão registrados por possuírem o ingrediente ativo 2,4D em sua formulação (ANVISA, 2007).

Foram descritos 66 casos de intoxicação por herbicidas fenoxiácidos entre 1962 e 1999 em que a maioria dos casos envolvia o 2,4-D (BRADBERRY et al., 2000). Além disso, um estudo realizado no Mato Grosso do Sul verificou-se que dos 880 casos de intoxicação ocupacional, ocorridos entre 1992 e 2000, 13,4\% envolviam herbicidas e, na maioria deles o principal causador foi o 2,4-D (RECENA et al., 2006).

Os efeitos de diferentes níveis de concentração do 2,4-D administrados em doses únicas ou continuados sobre alguns sistemas orgânicos animais, principalmente de cães e ratos, têm sido analisados (HANSEN; QUAIFE; HABERMANN, 1971; EPA, 1987; KELLY; GUIDOTTI, 1989; TSMAN, 1991; CHARLES et al., 1996a e 1996b; PAULINO; GUERRA; PALERMO-NETO, 1996; MATTSSON et al., 1997; GARABRANT; PHILBERT, 2002; AYDIN; ÖZDEMIR; UZUNÖREN, 2005; BUKOWSKA et al., 2008).

Em experimentos com ratos não houve mortalidade significativa de animais que receberam administração aguda (15 dias) e crônica (42 dias), tanto na dosagem de 2,5 quanto na de $5,0 \mathrm{mg} / \mathrm{kg} / \mathrm{dia}$ (MARGONATO; BATISTA; BARONI, 2003).

As pesquisas têm revelado que a dose tóxica do 2,4D para o ser humano, após ingestão, está em torno de 3 a $4 \mathrm{~g}$ e a dose letal é de $28 \mathrm{~g}$ (TSMAN, 1991). Já para ratos a dose letal é de $375 \mathrm{mg} / \mathrm{kg}$ de peso corpóreo (ALMEIDA; RODRIGUES, 1988), sendo considerado moderadamente tóxico para aves e não tóxicos para peixes (THOMPSON et al., 1984).
São sintomas da ingestão do 2,4-D pelo homem, queimação na língua e no esôfago, dor no peito, vômito, hemorragia gastrointestinal e gastrite aguda (BRADEBERRY et al., 2000; ABDOLLAHY et al., 2004). Efeitos neurotóxicos incluem coma, hiperreflexia, ataxia, alucinações, convulsões e paralisia (BRADEBERRY et al., 2000). As pesquisas que verificam a neurotoxicidade do 2,4-D têm sido voltadas para análises do sistema nervoso central (EPA, 1987; TSMAN, 1991; MATTSSON et al., 1997). O 2,4-D parece ser inibidor moderado da fosforilação oxidativa e tem ação tóxica direta sobre os músculos estriados esqueléticos e uma possível, porém discutida, ação tóxica sobre os nervos (TSMAN, 1991). Contudo, o mecanismo da neurotoxicidade do 2,4-D ainda não está esclarecido (BONGIOVANNI et al., 2007; BJORLING-POULSEN; ANDERSON; GRANDJEAN, 2008; KONJUH et al., 2008).

$\mathrm{Na}$ divisão própria do sistema nervoso autônomo, além do sistema simpático e parassimpático, atualmente é reconhecido o Sistema Nervoso Entérico (SNE) (STERNINI, 1988), como uma complexa rede de fibras nervosas e corpos celulares neuronais (SOUZA; FURLAN, 2001; FURNESS, 2006). Composto de interneurônios, neurônios sensoriais e neurônios motores, excitatórios e inibitórios, se estende ao longo de todo o tubo digestório (FURNESS, 2006).

O SNE é formado por diversos plexos, tendo como principais os plexos submucoso e mioentérico que atuam sobre os mecanismos da digestão e absorção de nutrientes, controlando a motricidade, fluxo sanguíneo e regulando primariamente a atividade secretomotora do sistema digestório (GABELLA, 1969; STERNINI, 1988; COSTA; BROOKES; HENNIG, 2000; KUTCHAI, 2000; SCHEMANN; NEUNLIST, 2004; PHILLIPS; POWLEY, 2007).

Alterações nos neurônios do plexo mioentérico são responsabilizadas por manifestações como anorexia, constipação, diarreia, perda de peso, vômitos, náusea, entre outras evidenciadas em diferentes condições experimentais como diabetes, (ZANONI et al., 2005; ALVES et al., 2006; CHANDRASEKHARAN; SRINIVASAN, 2007; SHOTTON; ADAMS; LINCOLN, 2007; PEREIRA et al., 2008; SILVERIO et al., 2009); envelhecimento (SANTER, 1994; JOHNSON et al., 1998; BRITTO MARI et al., 2008; GAGLIARDO et al., 2008); desnutrição (MOREIRA et al., 2008); desnutrição ao longo do envelhecimento (COWEN et al., 2000; SCHOFFEN et al., 2005; ARAÚJO et al., 2006); infecções (SUGAUARA et al., 2008, 2009); enterites e colites (BOYER et al. 2005, 2007; LOMAX et al., 2006) e carência protéica (ALVES et al., 2009), intoxicações (PEREIRA; STABILLE, 2006; CORREA et al., 2011; PEREIRA et al., 2013).

Uma vez que o 2,4-D tem ação sobre o sistema nervoso central é de se esperar que atue também sobre os neurônios do sistema nervoso entérico, já que entre as manifestações da intoxicação por 2,4-D encontram-se anorexia, 
irritação gastrointestinal, náuseas, vômitos e diarreia (EPA, 1987).

Sabendo dos efeitos neurotóxicos do ácido 2,4 diclorofenoxiacético o presente trabalho teve o objetivo de avaliar os efeitos do 2,4-D sobre os neurônios mioentéricos do duodeno de ratos por meio de análise quantitativa utilizando para tanto as técnicas histoquímicas de marcação da população neuronal mioentérica.

\section{Material e Métodos}

Esta pesquisa foi desenvolvida de acordo com os princípios éticos da experimentação animal, aprovada pelo Comitê de Ética em Pesquisa Envolvendo Experimentação Animal da UNIPAR (CEPEEA/UNIPAR), protocolo $n^{\circ}$ 22625/2011.

Para a realização deste estudo foram utilizados 20 ratos machos (Rattusnorvegicus), da linhagem Wistar e de 60 dias de idade, provenientes do Biotério da Universidade Estadual de Maringá.

Os animais foram mantidos em caixas plásticas individuais, em sala com ciclo de iluminação artificial controlado para 12 horas claro/12 horas escuro, a uma temperatura ambiente de $22^{\circ} \mathrm{C} \pm 2$, pelo período de 60 dias, e receberam ração comercial para roedores e água sem restrição.

Os animais foram distribuídos aleatoriamente em quatro grupos ( $\mathrm{n}=5 /$ grupo) .GC-I: grupo que serviu de controle para os animais que foram submetidos à ingestão de 2,4-D. e ao final do período experimental tiveram seus neurônios evidenciados pela técnica histoquímica de NADH diaforase. GC-II: grupo de animais que serviu de controle para os animais que foram submetidos à ingestão de 2,4-D. e ao final do período experimental tiveram seus neurônios evidenciados pela técnica histoquímica de NADPH diaforase.GE-I: NADH: grupo experimental que recebeu durante 60 dias $5 \mathrm{mg} / \mathrm{kg}$ de peso vivo/dia de 2,4-D diluído em $0,5 \mathrm{ml}$ de água via gavagem, e ao final do período experimental tiveram seus neurônios evidenciados pela técnica histoquímica de NADH. GE-II: NADPH: grupo experimental que recebeu durante 60 dias $5 \mathrm{mg} / \mathrm{kg}$ de peso vivo/dia de 2,4-D diluído em $0,5 \mathrm{ml}$ de água, via gavagem, e ao final do período experimental tiveram seus neurônios evidenciados pela técnica histoquímica de NADPH.

Após o período experimental de 60 dias e jejum de 12 horas, os animais foram submetidos à anestesia inalatória profunda com isoflurano e mortos por superexposição ao anestésico (HECKER; LAKE; DIFAZIO, 1983), para coleta do duodeno.

O duodeno obtido de 10 animais (GE-I e GC-I) foi lavado e preenchido com solução de Krebs (pH 7,3). O método de NADH-diaforase foi utilizado para evidenciar os neu- rônios mioentéricos, metabolicamente ativos, e gânglios nos preparados de membranas. Para tanto, o segmento foi lavado com solução de Krebs e incubado em meio de reação constituída de solução estoque de Nitro Blue Tetrazolium (NBT) conforme metodologia descrita por Gabella (1969).

O duodeno coletado de outros dez animais (GE-II e GC-II) foi lavado e preenchido com tampão fosfato (PB $\mathrm{pH} 7,4)$. O segmento foi então fixado em paraformaldeído por 30 minutos, imerso em Triton X-100 por 10 minutos e lavado três vezes (10 minutos cada lavagem) em PBS. Em sequência, o duodeno foi incubado para evidenciação dos neurônios nitrérgicos, em um meio de reação contendo Nitro Blue Tetrazolium (NBT), seguindo a metodologia descrita por Scherer-Singler et al. (1983).

Para a obtenção dos preparados de membrana, o duodeno foi seccionado ao longo da extensão da borda mesentérica e em seguida, foi microdissecado sob estereomicroscópio.

Os preparados de membrana obtidos dos duodenos submetidos aos procedimentos pertinentes às histoquímicas de NADH e NADPH-diaforase foram desidratados em série crescente de álcoois $(90 \%, 95 \%$ e absoluto), diafanizados por três imersões consecutivas em xilol e, em seguida, colocados entre lâmina e lamínula de vidro com resina sintética.

Os preparados de membrana foram destinados à quantificação dos neurônios mioentéricos.

Para a quantificação neuronal por área $\left(\mathrm{mm}^{2}\right)$, o preparado de membrana do duodeno obtido de cada animal foi visualizado ao microscópio de luz Olympus BX40, com aumento de 40X. A imagem verificada no microscópio foi capturada por câmera digital de alta resolução e transferida para o computador. Os neurônios foram quantificados em 80 campos microscópicos por preparado de membrana.

A análise estatística foi realizada utilizando o Programa estatístico Sisvar (Versão 5.0). Os dados foram submetidos à análise de variância ANOVA (one-way) e ao pós-teste T de Student. Os resultados foram descritos como média \pm erro padrão, tendo sido adotado o nível de significância de 5\%.

A área fornecida pela objetiva de $40 x$ previamente mensurada foi de $0,096 \mathrm{~mm}^{2}$, sendo que os 80 campos representaram a área total de $7,68 \mathrm{~mm}^{2}$ de duodeno, permitindo a determinação da densidade neuronal por $\mathrm{mm}^{2}$ de duodeno.

\section{Resultados}

Foram analisados os dados referentes ao peso inicial, ao peso após 20 dias, após 40 dias e ao final do período experimental (após 60 dias) de todos os grupos (tabela 1). O peso corporal dos animais não diferiu estatisticamente $(\mathrm{P}>0,05)$ entre os grupos.

Tabela 1: Média do peso corporal (g) inicial, após 20 dias, após 40 dias e ao final do período experimento dos grupos controle I (GCI-NADH), grupo controle II (GCII-NADPH), grupo experimental I (GEI - NADH) e grupo experimental II (GEII - NADPH)

\begin{tabular}{lllll}
\hline \multicolumn{1}{c}{ Parâmetros } & \multicolumn{1}{c}{ GCI-NADH } & \multicolumn{1}{c}{ GCII-NADPH } & \multicolumn{1}{c}{ GEI-NADH } & \multicolumn{1}{c}{ GEII-NADPH } \\
\hline Peso inicial & $283,2 \pm 9,57$ & $242,2 \pm 4,43$ & $281,8 \pm 3,34$ & $238,6 \pm 3,64$ \\
Após 20 dias & $399,6 \pm 34,18$ & $341,6 \pm 10,85$ & $384,4 \pm 26,17$ & $337,2 \pm 11,94$ \\
Após 40 dias & $472,8 \pm 39,49$ & $422,2 \pm 25.26$ & $448 \pm 43,20$ & $394,8 \pm 22,2$ \\
Peso final & $521,6 \pm 50,35$ & $451,4 \pm 40,51$ & $503,6 \pm 51,77$ & $442,8 \pm 30,35$ \\
\hline
\end{tabular}

Não houve diferenças significativas pela ANOVA. 
$\mathrm{Na}$ análise por microscopia de luz, os neurônios mioentéricos NADH-diaforase positivos (Figura 1a) e NADPH-diaforase positivos (Figura 1c) foram encontrados or-
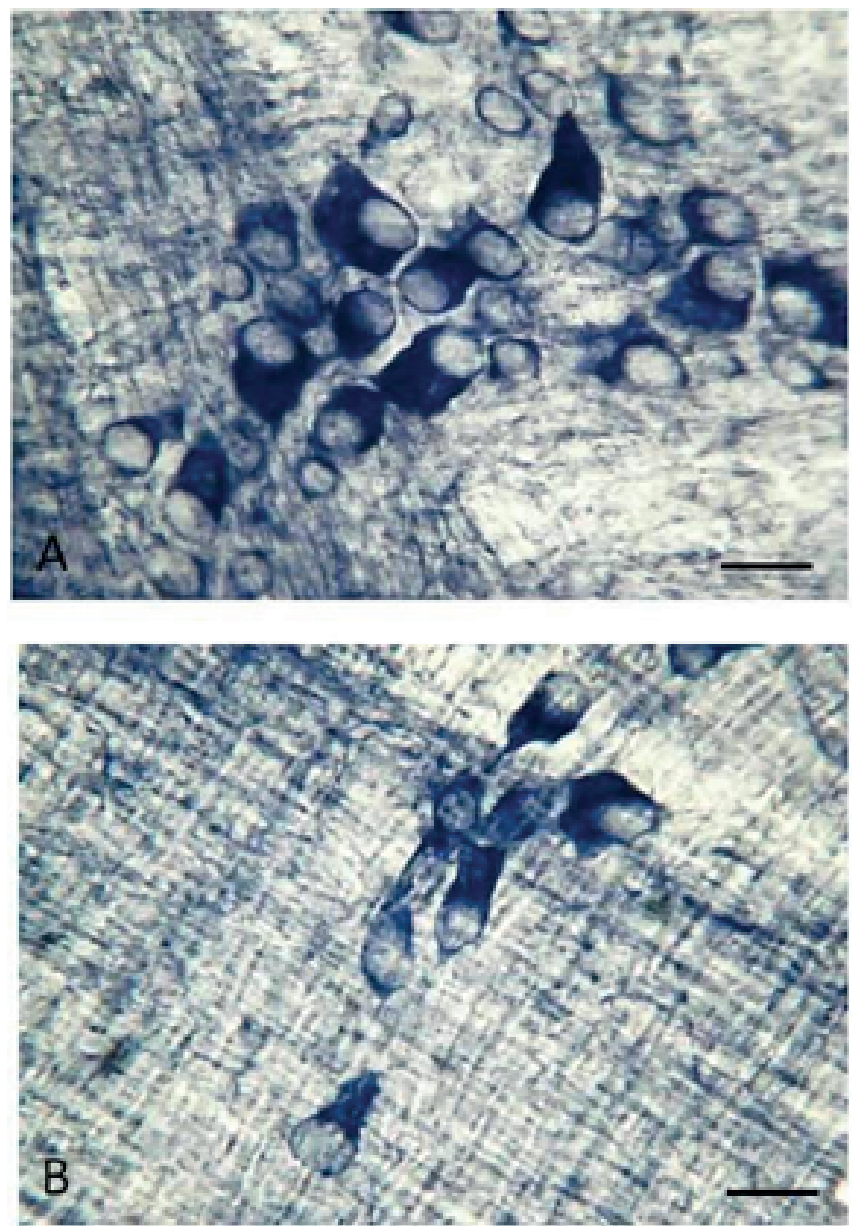

Figura 1: Fotomicrografia de preparados de membrana do duodeno de ratos evidenciando neurônios do plexo mioentérico dos grupos controle (A, C) e experimental (B, D) marcados por diferentes técnicas: em A e B neurônios corados pela histoquímica da NADH-diaforase; em C e D neurônios corados pela histoquímica da NADPH-diaforase. Barra=100 $\mu \mathrm{m}$

A quantificação de neurônios evidenciados pelas técnicas histoquímica de NADH-d (neurônios metabolicamente ativos) e pela NADPH-d (neurônios inibitórios) diferiu estatisticamente $(\mathrm{P}<0,05)$ entre os grupos controle e experimental de cada técnica e também entre as técnicas (Tabela 2).

Tabela 2: Densidade Neuronal expressa em média e desvio padrão dos neurônios do duodeno de ratos dos grupos GCI, GEI-NADH, GCII E GEII-NADPH.

\begin{tabular}{ccc}
\hline \multicolumn{3}{c}{ Densidade neuronal expressa em $\mathbf{~ m m}^{\mathbf{2}}$} \\
\hline Grupos & NADHd + & NADPH+ \\
\cline { 2 - 3 } GC $(\mathrm{n}=5)$ & $84,3 \pm 18,30^{\mathrm{a}}$ & $36,11 \pm 5,46^{\mathrm{a}}$ \\
GE $(\mathrm{n}=5)$ & $64,14 \pm 4,86^{\mathrm{b}}$ & $45,33 \pm 6,48^{\mathrm{b}}$ \\
\hline
\end{tabular}

Médias seguidas por letras diferentes na mesma coluna diferem estatisticamente pelo teste T de Student $(\mathrm{P}<0,05)$

\section{Discussão}

Segundo Garabrant e Philbert (2002), a dose de $1 \mathrm{mg} / \mathrm{kg} /$ dia foi determinada como a dose que não promove efeitos tóxicos (NOAEL - no observed adverse effectlevel) renais, hematológicos e hepáticos em ratos, contudo, poucos

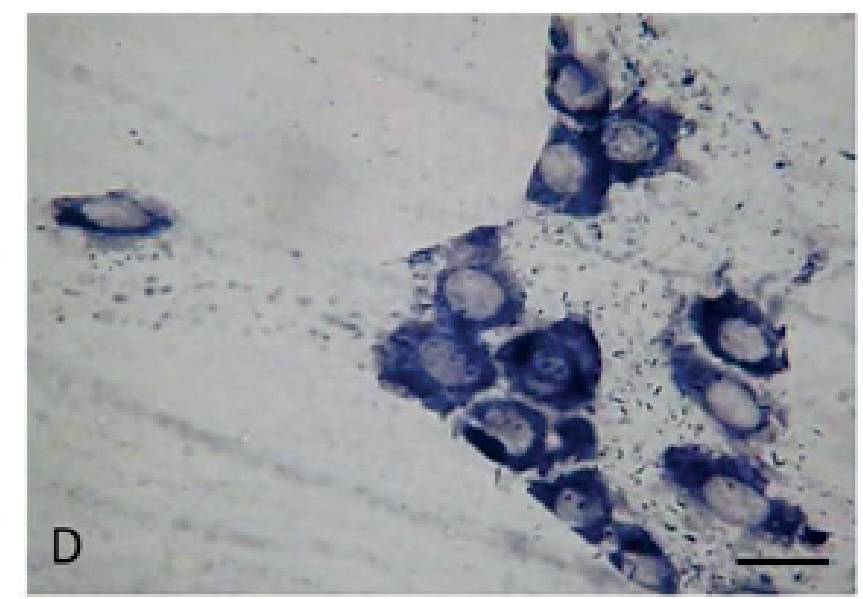

ganizados em gânglios e interconectados por feixes de fibras nervosas demonstrando organização espacial característico para a espécie.

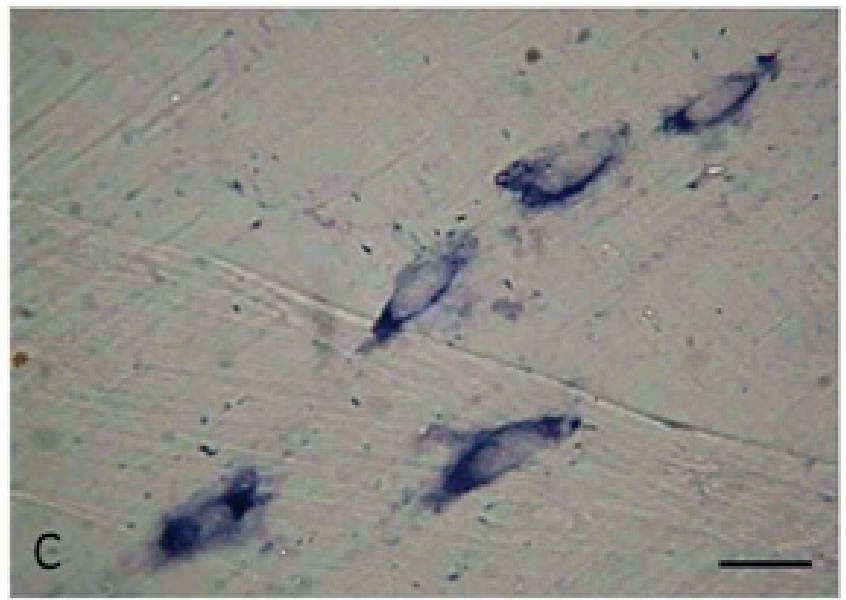

são os relatos dos efeitos tóxicos do 2,4D sobre os neurônios mioentéricos, por este motivo optou-se por verificar os efeitos da ingestão da dose de $5 \mathrm{mg} / \mathrm{kg} /$ dia, dose semelhante aquela utilizada por Correa et al. (2011) para o íleo e por Pereira et al. (2013) para o duodeno de ratos em um período experimental de 15 dias.

Ao longo do experimento foram observadas alterações comportamentais dos animais, como irritabilidade durante o manuseio, sintoma característico da intoxicação por 2,4D (EPA, 1987; SCHVARTSMAN, 1991; MATTSSON et al., 1997), porém não houve morte dos ratos.

O peso dos animais nos diferentes grupos não diferiu estatisticamente $(\mathrm{P}>0,05)$ em nenhum período do tratamento, indicando que a dosagem de $5 \mathrm{mg} / \mathrm{kg} /$ diade $2,4-\mathrm{D}$, durante 60 dias, não promove alterações no peso corporal de ratos. Resultados semelhantes foram observados em trabalhos nas doses de 2,5 e $5 \mathrm{mg} / \mathrm{Kg} /$ peso em experimentos conduzidos por um período de 15 dias sobre o duodeno e o jejuno de ratos (PEREIRA; STABILLE, 2006; NANNI, 2010; CORREA et al., 2011, PEREIRA, et al., 2013). Doses superiores variando entre 15 e $70 \mathrm{mg} / \mathrm{kg} /$ dia também não interferiram no ganho de peso de ratos (STURTZ et al., 2006; KONJUH et al., 2008).

A organização ganglionar dos neurônios NADH e 
NADPH, vistos neste trabalho, estão de acordo com o descrito na literatura para neurônios do plexo mioentérico (ZANIN et al., 2001; FURNESS, 2006; PEREIRA et al., 2013) evidenciando que na dose utilizada no período de 60 dias, não promove alterações desta distribuição espacial. Os neurônios mioentéricos foram encontrados entre as camadas longitudinal e circular da túnica muscular como já observado para o intestino delgado de ratos e outros animais (MOLINARI et al., 1994; STABILLE; LIMA; GERMANO, 1999; MIRANDA-NETO et al., 2001; HANSEN, 2006; CORREA et al., 2011; PEREIRA, et al., 2013).

Os neurônios evidenciados pela NADH-diaforase, encontram-se em maior densidade no grupo controle em comparação ao experimental, esta diminuição na quantidade de neurônios NADHd+em animais submetidos a ingestão de 2,4-D, sugere um possível efeito neurotóxico deste herbicida sobre os neurônios do sistema nervoso entérico, que segundo Miranda-Neto et al. $(2001 ; 2005)$ os neurônios corados pela histoquímica da NADH-diaforas e evidencia apenas neurônios metabolicamente ativos, sendo esta condição confirmada quando utilizadas técnicas de coloração da população total em comparação a NADH, onde a marcação neuronal foi de cerca de $80 \%$ da população total (YOUNG et al., 1993).

Quanto ao mecanismo envolvido na diminuição dos neurônios mioentéricos NADH-d+ poderia ser atribuído ao estresse oxidativo, visto quese acredita que o estresse oxidativo seja um evento secundário no processo patogênico incluindo as intoxicações pelos herbicidas derivados do 2,4D (BAYNES; THORPE, 1999, BONGIOVANNI et al., 2007). $\mathrm{O}$ estresse oxidativo ocorre num sistema celular quando o oxigênio reativo leve à danos nas membranas ou partes internas das células, modifique proteínas, como o colágeno e a elastina ou provoque mudanças nas moléculas de DNA (GUTTERIDGE; HALLIWELL, 1992). Proteínas que são danificadas por estresse oxidativo têm diminuição da atividade biológica, levando à perda do metabolismo de energia, sinalização celular, transporte e, em última instância, pode conduzir à morte celular (VINCENT et al., 2004).

Pereira e Stabille (2006) observou uma redução de $21,98 \%$ e $10,47 \%$ no número de neurôniosdos grupos tratados com 5,0 e 2,5mg/kg de 2,4-D. A diminuição do número de neurônios NADHd+ também foi observada em ratos submetidos a outras condições adversas de saúde, como por exemplo, no diabetes (CLEBIS et al., 2004). O estresse oxidativo seguido dos danos oxidativos nos tecidos, são pontos finais comuns de doenças crônicas, como aterosclerose, diabetes, artrite reumatoide e também por doenças hepáticas e renais provocadas pelas intoxicações (BAYNES; THORPE, 1999, BONGIOVANNI, et al., 2007).

Neurônios mioentéricos nitrérgicos podem ser evidenciados pelo método histoquímico da enzima nicotinamida adenina dinucleotídeo fosfato diaforase (NADPH-diaforase) (SCHERER-SINGLER et al., 1983), em cuja subpopulação neuronal encontra-se em maior densidade no grupo experimental. São neurônios que sintetizam o óxido nítrico (NO) como neurotransmissor, que no sistema nervoso entérico funciona como inibitório para o músculo liso do tubo digestório (BELAI et al., 1992).

Este aumento da população nitrérgica, sugere maior resistência destes neurônios à morte celular (SANTER, 1994), porpossuírem mecanismos de defesa contra danos causados por radicais livres (COWEN et al., 2000), contudo, cabe destacar que o NO tem ação benéfica e prejudicial nas células (WALLACE; MILLER, 2000; DANIELS et al., 2005), mesmo não tendo sido este, o enfoque desta pesquisa.

Correa et al. (2011) registrou um aumento, apesar de não significativo $(\mathrm{P}>0,05)$, na expressão da subpopulação de neurônios NADPHdp no jejuno de ratos do grupo experimental, em experimento conduzido por um período de 15 dias e na dosagem de $5 \mathrm{mg} / \mathrm{kg} / \mathrm{dia}$ de $2,4-\mathrm{D}$, resultados semelhantes foram descritos para o duodeno em condições experimentais semelhantes (PEREIRA, et al., 2013). A diferença dos resultados pode ser atribuída, a maior exposição dos ratos ao 2,4D, que foi de 60 dias, impondo neste caso, a uma maior condição de alterações metabólicas, nos neurônios nitrérgicos do grupo experimental. Segundo Paulino et al. (1996) os sinais de intoxicação pelo 2,4D são dose e tempo dependentes em animais de laboratórios, assim também pode se esperar que sua detecção tecidual também o seja.

Há relatos de que os neurônios NADPHdp são resistentes ao envelhecimento, ao diabetes e a outras condições adversas (DAWSON et al., 1991; BELAI et al., 1995; JOHNSON et al., 1998; FURLAN et al., 2004). São também mais resistentes às condições que determinam morte celular, por apresentarem possível efeito defensivo antioxidante (BOLAÑOS et al., 1997), porém não são absolutamente imunes às alterações fisiológicas destes processos, podendo progressivamente tornarem-se comprometidos (PHILLIPS; KIEFFER; POWLEY, 2003).

O mecanismo de ação do 2,4-D ainda não foi totalmente compreendido (BONGIOVANNI et al., 2007), ressalta-se porém que o 2,4-D seja um inibidor da fosforilação oxidativa (SCHVARTSMAN, 1991; BRADBERRY et al., 2000). O uso extensivo deste herbicida motiva pesquisas sobre suas propriedades e efeitos sobre os organismos vivos e o meio ambiente. (AMARANTE-JUNIOR, 2002).

\section{Conclusão}

Os resultados obtidos nessa pesquisa permitiram verificar que a ingestão de $2,4 \mathrm{D}$ na dose de $5 \mathrm{mg} / \mathrm{kg} / \mathrm{dia}$, por 60 dias, interfere na expressão neuronal, levando a diminuição na densidade dos neurônios metabolicamente ativos, sugerindo provável efeito tóxico do $2,4 \mathrm{D}$, contudo o aumento da reatividade dos neurônios nitrérgicos, pode indicar maior resistência desta subpopulação à ação deste herbicida.

\section{Agradecimentos}

Os autores agradecem a Fundação Araucária pela concessão da bolsa PEBIC para o primeiro autor, e a UNIPAR pelo suporte financeiro para o desenvolvimento da Iniciação Científica.

\section{Referências}

ABDOLLAHI, M. et al. Pesticides and oxidative: a review. Medical Science Monitor, Hicksville, NY, v. 10, n. 6, p. 141-147, 2004.

ALMEIDA, F. S.; RODRIGUES, B. N. Guia de

Herbicidas, 2. ed. Londrina: Autores, 1988. p. 175-185. 
ALVES, A. M. et al. Morphoquantitative aspects of nadhdiaphorasemyenteric neurons in the ileum of diabetic rats treated with acetyl-L-carnitine. Anatomia, Histologia, Embryologia, Berlim, v. 35, n. 1, p. 13-18, 2006.

AMARANTE-JUNIOR, O. P. et al. Revisão

das propriedades, usos e legislação do ácido 2,4 diclorofenoxiacético (2,4-D). Cadernos de Pesquisa, São Luís, v. 13, n. 1, p. 60-70, 2002.

ANVISA. Agência Nacional de Vigilância Sanitária. Disponível em: <www. anvisa. gov.br/ toxicologia/ monografias/ p 07. Pdf>. Acesso em: 17dez. 2013.

ARAÚJO, A. E. J. et al. Quantitative study of the myenteric plexus of the descending colon of young rats subjected to intense protein deficiency. International Journal of Morphology, Temuco, v. 24, p. 591-597, 2006.

AYDIN, H.; ÖZDEMIR, N.; UZUNÖREN, N. Investigation of the accumulation of 2,4 dichlorophenoxyacetic acid (2,4D) in rat kidneys. Forensic Science International, Turku, v. 153 , p. 53-57, 2005.

BAYNES, J. W.; THORPE, S. R. Role of Oxidative Stress in Diabetic Complications. A New Perspective on an Old Paradigm. Diabetes, Alexandria, v. 48, n. 1, p. 1-9, 1999.

BELAI, A. et al. Colocalization of nitric oxide synthase and NADPH-diaphorase in the myoenteric plexus of the rat gut. Neuroscience Letter, Amsterdam, v. 143, p. 60-64, 1992.

BELAI, A.; COOPER, S.; BURNSTOCK, G. Effect of age on NADPH-diaforase-containing myenteric neurons of rat ileum and proximal colon. Cell and Tissue Research, Berlim, v. 279, p. 379-383, 1995.

BERNARD, H. et al. Assessment of herbicide leaching risk in two tropical soils of Reunion island (france). Journal of Environmental Quality, Madison, v. 2, n. 34, p. 53-54, 2005.

BJORLING-POULSEN, M.; ANDERSON, H. R.; GRANDJEAN, P. Potential developmental neurotoxicity of pesticides used in Europe. Environmental Health, London, v. 7, p. 1-50, 2008.

BOLAÑOS, J. P. et al. Nitric oxide-mediated mitochondrial damage in the brain: mechanisms and implications for neurodegenerative diseases. Journal of Neurochemistry, New York, v. 68, p. 2227-2240, 1997.

BONGIOVANNI, B. et al. Melatonin decreases the oxidative stress produced by 2,4-dichlorophenoxyacetic acid in rat cerebellar granule cells. Neurotoxicology Research, Little Rock, v. 11, p. 93-99, 2007.

BOYER, L. et al. Myenteric plexus injury and apoptosis in experimental colitis. Autonomic Neuroscience: Basic and Clinical, London, v. 117, p. 41-53, 2005.
BOYER, L. et al. Differential responses of VIP and nitrergicneurons in pediatric patients with Crohn's disease. Autonomic Neuroscience: Basic and Clinical, London, v. 134, p. 106-114, 2007.

BRADBERRY, S. M. et al. Mechanisms of toxicity, clinical features and management of herbicide poisoning: A review. Clinical Toxicology, Salt Lake City, Utah, v. 38, p. 111122,2000

BRASIL. Ministério da Saúde, Secretaria de atenção à saúde, Departamento de ações programáticas estratégicas, Área técnica de saúde do trabalhador, Diretrizes para Atenção Integral à Saúde do trabalhador de Complexidade Diferenciada. Protocolo de Atenção à saúde dos Trabalhadores Expostos a agrotóxicos, 2006.

BRITTO MARI, R. et al. Effects of exercise on the morphology on the myenteric neurons of the duodenum of wistar rats during the ageing process. Anatomia, Histologia, Embryologia, Berlim, v. 37, p. 289-295, 2008.

BUKOWSKA, B. et al. Phenoxyherbicides induce production of free radicals in human erythrocytes: oxidation of dichlorohydrofluorescine and dihydrorhodamine 123 by 2,4-D-Na and MCPA-Na. Food and Chemical Toxicology, Andover, v. 46, n. 1, p. 359-367, 2008.

CHANDRASEKHARAM, B.; SRINIVASAN, S. Diabetes and enteric nervous system. Journal of Neurogastroenterology and Motillity, Rochester, v. 19, p. 6-18, 2007.

CHARLES, J. M. et al. Chronic dietary toxicity/ oncogenicity studies on 2,4-dichlorophenoxyacetic acid in rodents. Fundamental and Applied Toxicology, California, v. 33, p. 166-172, 1996a.

CHARLES, J. M. et al. Comparative subchronic studies on 2,4-dichlorophenoxyacetic acid, amine and ester in rats. Fundamental and Applied Toxicology, California, v. 33, p. $161-165,1996 b$.

CLEBIS, N. K. et al. Avaliação quantitativa e morfometrica dos neurônios mioentéricos da região aglandular do estômago de ratos com diabetes Mellitus induzido por estreptozootocina e suplementados com ácido ascórbico.

Arquivo de Ciências da Saúde Unipar,Umuarama, v. 8, n. 2, p. 87-93, 2004.

CORREA, O. P. et al. Effects of the ingestion of 2,4 Dichlorophenoxyacetic acid on jejunalmyenteric neurons in rats. Journal of Morphological Science, São Paulo, v. 28, n. 2, p. 104-112, 2011.

COSTA, M.; BROOKES, S. J. H.; HENNIG, G. W. Anatomy and physiology of the enteric nervous system. Gut, London, v. 47, p. 15-19, 2000.

COWEN, T. et al. Restricted diet rescues rat enteric motor neurones from age related cell death. Gut, London, v. 47, p. 
653-660, 2000.

DANIELS, I. et al. Evaluated expression of NOS mRNA and protein in celiac disease. Clinica Chemica Acta, San Francisco, CA, v. 356, p. 134-142, 2005.

DAWSON, T. M. et al. Nitric oxide synthase and neuronal NADPH diaphorase are identical in brain and peripheral tissues. Proceedings of the National Academy of Science, Stanford, v. 8, p. 7797-7801, 1991.

EPA, Consumer Factsheet on: 2,4 D. Office of water Regulation and standards, 2005.

FURLAN, M. M. D. P. et al. Resposta dos neurônios mioentéricos do duodeno de ratos ao diabetes de curto prazo. Arquivo de Ciências da Saúde Unipar, Umuarama, v. 8, n. 2, p. 95-99, 2004.

FURNESS, J. B. The enteric nervous system. Malden: Blackwell Publishing, 2006.

GABELLA, G. Detection of nerve cells by histochemical technique. Experientia, Basel, v. 25, p. 218-219, 1969.

GAGLIARDO, K. M. et al. Exercise reduces inhibitory neuroactivity and protects myenteric neurons from agerelated neurodegeneration. Autonomic Neuroscience: Basic and Clinical, London, v. 141, p. 31-37, 2008.

GARABRANT, D. H.; PHILBERT, M. A. Review of 2,4-dichlorophenoxyacetic acid (2,4-D) epidemiology and toxicology. Critical Reviews in Toxicology, London, v. 32, p. 233-257, 2002.

GUTTERIDGE, J. M.; HALLIWELL, B. Comments on review of free radicals in biology and medicine. Free Radical Biology \& Medicine, New York, v. 12, n. 1, p. 93-95, 1992.

HANSEN, M. B. The enteric Nervous System II: Gastrointestinal. Journal of Pharmacological and Toxicological Methods, New York, v. 92, p. 105-113, 2006.

HANSEN, W. H.; QUAIFE, M. L.; HABERMANN, R. T. Chronic toxicity of 2,4-dichlorophenoxyacetic acid in rats an dogs. Toxicology and Applied Pharmacology, Albuquerque, v. 20, p. 122-129, 1971.

HECKER, B. R.; LAKE, C. L.; DIFAZIO, C. A. The decrease of the minimum alveolar anesthetic concentration produced by sulfentanil in rats. Anesthesia \&Analgesia, Baltimore, v. 62, p. 987-90, 1983.

JOHNSON, R. J. R. et al. The effects of age on the overall population and on sub-populations of myenteric neurons in the rat small intestine. Journal of Anatomy, London, v. 192, p. 479-88, 1998.

KELLY, S. J.; GUIDOTTI, T. L. Phenoxyacetic acid herbicides and chlorophenols and the etiology of lymphoma and soft-tissue neoplasms. Public Health Reviews, California, v. 17, p. 1-37, 1989.

KONJUH, C. et al. Neonatal hypomyelination by the herbicide 2,4-dichlorophenoxyacetic acid. Chemical and ultrastructural studies in rats. Toxicological Science, Reston, v. 104, n. 2, p. 332-340, 2008.

KUTCHAI, H. C. Motilidade gastrointestinal. In: BERNE, R. M.; LEVY, M. N. Fisiologia. Rio de Janeiro: Guanabara Koogan, 2000. p. 555-5.

LOMAX, A. E. et al. Effects of gastrointestinal inflammation on enteroendocrine cells and enteric neural reflex circuits. Autonomic Neuroscience: Basic and Clinical, London, v. 126, p. 250-257, 2006.

MARGONATO, F. B.; BATISTA M. R.; BARONI, E. A. Efeito do agrotóxico 2,4-D (ácido 2,4-diclorfenoxiacético) na morfologia e função renal de ratos Wistar. In: ENCONTRO ANUAL DE INICIAÇÃO CIENTIFICA, 6., 2003. Maringá. Anais do encontro anual de iniciação cientifica, Maringá: UEM, 2003

MATTSSON, J. L. et al. Single-dose and chronic dietary neurotoxicity screening studies on 2,4-dichlorophenoxyacetic acid in rats. Fundamental and Applied Toxicology, California, v. 40, p. 11-119, 1997.

MIRANDA NETO, M. H. et al. Regional differences in the number and type myenteric neurons of the ileum of rats. Arquivos de Neuropsiquiatria, São Paulo, v. 59, p. 54-59, 2001.

MIRANDA-NETO, M. H. et al. Morphometric and quantitative evaluation of the NADH-diaphorase positive myenteric neurons of the jejunum of streptozotocin-diabetic rats supplemented with acetyl-L-carnitine. Anatomia, Histologia, Embryologia, Berlim, v. 34, n. 3, p. 154-158, 2005.

MOLINARI, S. L. et al. Estudo morfológico do plexo mioentérico do estomago do pato (Anassp). Revista UNIMAR, Maringá, v.16, n. 2, p. 419-426, 1994.

MOREIRA, N. M. et al. Quantitative analysis of the neurons from the myenteric plexus in the ileum of rats submitted to severe proteic deficiency. Arquivos de Neuropsiquiatria, São Paulo, v.26, n. 2, p. 242-245, 2008.

NANNI,W. Avaliação morfoquantitativa de neurônios mientéricos do cólon proximal de ratos tratados com o herbicida ácido 2,4-diclorofenoxiacetico. 2010. 74f. Dissertação (Mestrado em Ciência Animal) - Universidade Paranaense - UNIPAR, Umuarama, 2010.

PAULINO, C. A.; GUERRA, J. L.; PALERMO-NETO, J. Acute, subchronic and chronic 2,4-dichlorophenoxyacetic acid (2,4-D) intoxication in rats. Veterinary and Human Toxicology, Manhattan, v. 38, n. 5, p. 348-352, 1996. 
PEREIRA, A. P. C.; STABILLE, S. R. Efeitos da ingestão do herbicida ácido 2,4-diclorofenoxiacético sobre os neurônios mioentéricos do duodeno de ratos (Rattusnorvegicus): análises morfométrica e quantitativa. Revista UNINGÁ, Maringá, n. 9, p. 127-142, 2006.

PEREIRA, J. N. B. et al. Alterations in the duodenum myenteric neurons of Wistar rats after ingesting of 2,4 dichlorophenoxyacetic acid. Journal of Morphological Science, São Paulo, v. 30, n. 1, p. 28-32. 2013.

PEREIRA, R. V. et al. Vitamin E supplementation in rats with experimental diabetes mellitus: analysis of myosin- $\mathrm{V}$ and NOS imunoreactivemyenteric neurons from terminal ileum. Journal of Molecular Histology, Dordrecht, v. 39, n. 6 , p. 595-603, 2008.

PHILLIPS, R. J.; KIEFFER, E. J.; POWLEY, T. L. Aging of the myenteric plexus: neuronal loss is specific to cholinergic neurons. Autonomic neuroscience, Amsterdam, v. 106, n. 2, p. 69-83, 2003.

PHILLIPS, R. J.; POWLEY, T. L. Innervation of the gastrointestinal tract: patterns of aging. Autonomic Neuroscience: Basic and Clinical, London, v. 136, p. 1-19, 2007.

RECENA, M. C. P. et al. Acute poisoning with pesticides in the state of Mato Grosso do Sul, Brazil. Science of the total envirommental, Barcelona, v. 37, p. 88-95, 2006.

RODRIGUES, M. V. N.; SERRA, G. E. Pesticidas, 1996. $99 \mathrm{p}$.

SANTER, R. M. Survival of the population of the NADPHdiaphorase stained myenteric neurons in the small intestine of aged rats. Journal of the Autonomic Nervous System, Amsterdam, v. 49, p. 115-12, 1994.

SCHEMANN, M.; NEUNLIST, M. The human enteric nervous system. Journal of Neurogastroenterology and Motillity, Rochester, v. 16, p. 55-59, 2004.

SCHERER-SINGLER, U. et al. Demonstration of unique population of neurons with nadph-diaphorasehistochemistry. Journal of Neuroscience Methods, UK,v. 9, n. 3, p. 229234, 1983.

SCHOFFEN, J. P. F. et al. Effects of hypoproteic diet on myosin- $\mathrm{V}$ imunostainedmyenteric neurons and the proximal colon of aging rats. Autonomic Neuroscience: Basic and Clinical., London, v. 83, p. 122-77, 2005.

SCHVARTSMAN, S. Intoxicações agudas.4. ed. São Paulo: Sarvier, 1991. p. 266-267.

SHOTTON, H. R.; ADAMS, A.; LINCOLN, J. Effect of aminoguanididine treatment on diabetes-induced changes in myenteric plexus of rat ileum. Autonomic Neuroscience, Amsterdam, v. 132, p. 16-26, 2007.
SILVERIO, S. M. et al. Effects of acorbic acid supplementation in ileum myenteric neurons streptozotocininduced diabetic rats. Revista Pesquisa Veterinária Brasileira, Seropédica, Rio de Janeiro, v. 29, n. 4, p. 295 302, 2009.

SOUZA, J. A.; FURLAN, M. M. D. P. Avaliação morfométrica de neurônios mioentéricos do duodeno de ratos (Rattusnorvegicus) adultos normais e com diabetes experimental. Arquivos de Ciências da Saúde Unipar, Umuarama v. 5, n. 2, p. 141-147, 2001.

STABILLE, S. R.; LIMA, M. A.; GERMANO, R. M. Morphoquantitative characteristics of myenteric neurons of the terminal segment of the intestine of Cyprinuscarpio (Linnaeus, 1758) (Osteichthyes, Cyprinidae). Acta Scientiarum, Maringá, v. 16, n. 1, p 39-44, 1999.

STERNINI, C. Structural and chemical organization of the myoenteric plexus. Annual Review of Physiology, California, v. 50, p. 81-93, 1988.

STÜRTZ, N. et al. Detection of 2,4-dichlorophenoxyacetic acid in rat milk of dams exposed during lactation and milk analysis of their major components. Food and Chemical Toxicology, New York, v. 44, p. 8-16, 2006.

SUGAUARA, E. Y. Y. et al. Alterations on the myenteric plexus of the ileum and the descending colon caused by Toxoplasma gondii (genotype III). Arquivos de neuropsiquiatria, São Paulo, v. 66, n. 3, p. 516-523, 2008.

SUGAUARA, E. Y. Y. et al. Hypertrophy of the neurons in the ileum of rats infected with cysts of Toxoplasma gondii (genotype II). Acta Scientiarum Biological Science, Maringá, v. 31, n. 2, p. 195-201, 2009.

THOMPSON, D. G. et al. Persistence of (2,4-dichlorophenoxy) acetic acid and 2- (2,4dichlorophenoxy) propionic acid in agricultural and forest soils of northern and southern Ontario. Journal of Agricultural and Food Chemistry, Washington, v 32, n. 3, p 578-581, 1984.

TSMAN, S. S. Intoxicações agudas. 4.ed. São Paulo: Sarvier, 1991. p. 266-267.

UNITED STATES ENVIROMENTAL PROTECTION AGENCY -EPA.The risk assessment guidelines of 1986. Office of health and environmental assessment. Washington: DC. 1987.

VINCENT, A. M. et al. Oxidative stress in the pathogenesis of diabetic neuropathy. Endocrine Reviews, Stanford, v. 25, n. 4, p. 612-628, 2004.

WAITE, D. T. et al. Environmental concentrations of agricultural herbicides: 2,4-D and Triallate. Journal of Environmental Quality, Madison, v. 31, p. 129-144, 2002.

WALLACE, J. L.; MILLER, M. J. Nitric oxide in 
mucosal defense: a little goes a long way. Journal of

Gastroenterology, Philadelphia, v. 119, p. 512-520, 2000.

YOUNG, H. M. et al. Total numbers of the neurons in myenteric ganglia of the guinea-pig small intestine. Cell and Tissue Research, Freiburg, v. 272, p. 197-200, 1993.

ZANIN, S.M. et al. Densidade dos neurônios mioentéricos NADH-diaforase positivos do jejuno de ratos (Rattusnorvegicus). Arquivos de Ciências daSaúde Unipar, Umuarama, v. 5, n. 1, p. 3-8, 2001.

ZANONI, J. N. et al. Effects of supplementation with ascorbic acid for a period of 120 days on the myosin- $\mathrm{V}$ and nadph-d positive myenteric neurons of the ileum of rats.

Anatomia Histologia, Embryologia, Berlim, v. 34, p. 149$53,2005$.

Recebido em: 21/12/2013

Aceito em: 20/08/2014 